Int. J. Electrochem. Sci., 14 (2019) 4483 - 4495

\title{
Bismuth Nano-Flower Modified CPE for Anodic Stripping Voltammetry Detection of Cd(II)
}

\author{
Yuyuan Liu ${ }^{1}$, Jie Liu ${ }^{1}$, Qianli Zhang ${ }^{1, *}$, Jie Wei ${ }^{1}$, Guiyun Xu \\ ${ }^{1}$ College of Chemistry and Material Engineering, Jiangsu Key Laboratory for Environment Functional \\ Materials, Suzhou University of Science and Technology, Suzhou, 215009, China \\ ${ }^{2}$ Shandong Key Laboratory of Biochemical Analysis; College of Chemistry and Molecular \\ Engineering, Qingdao University of Science and Technology, Qingdao 266042, PR China \\ *E-mail: zqlmhb@163.com
}

doi: $10.20964 / 2019.05 .34$

Received: 7 January 2019 / Accepted: 2 March 2019 / Published: 10 April 2019

Bismuth (Bi) nano-needle, nano-rod, nano-sheet, and nano-flower were synthesized using a facile onestep solvothermal method by adjusting the volume of ethylenediamine (EDA). The prepared Bi nanoparticles were characterized by scanning electron microscope (SEM) and X-ray diffraction (XRD) analysis and their formation mechanism was discussed. Bi nano-particle modified carbon paste electrode (CPE) combined with differential pulse anodic stripping voltammetry (DPASV) was developed for the determination of $\mathrm{Cd}$ (II). It was shown that $\mathrm{Bi}$ nano-flower modified electrode displayed the highest detection sensitivity on account of its particular three-dimensional structures. The stripping peak currents of Cd (II) at "bulk" and "drop coating" Bi nano-flower modified CPEs were compared with those of Bi film modified CPEs using "in-situ" and "out-situ" plating methods. The optimized detection conditions were 5\% Bi nano-flower bulk modified $\mathrm{CPE}$ at $\mathrm{pH} 4.4$, deposition potential of $-1.2 \mathrm{~V}$ (vs. $\mathrm{AgCl} / \mathrm{Ag}$ ) and deposition time of $200 \mathrm{~s}$. The linear range, sensitivity and detection limit were $0.05-1 \mu \mathrm{M}, 27.5 \mu \mathrm{A} / \mu \mathrm{M}$, and $2.4 \mathrm{nM}$, respectively. The proposed Bi nano-flower bulk modified CPE was conveniently usable in the trace $\mathrm{Cd}$ (II) analysis for environmental water samples with the recovery rang of $91.1 \%-105.6 \%$.

Keywords: Bi nano-flower particles, CPE, Differential pulse anodic stripping voltammetry, Cd (II) trace detection

\section{FULL TEXT}

(C) 2019 The Authors. Published by ESG (www.electrochemsci.org). This article is an open access article distributed under the terms and conditions of the Creative Commons Attribution license (http://creativecommons.org/licenses/by/4.0/). 